\title{
An interactive program for observation and analysis of human behavior in a long-term continuous laboratory
}

\author{
DANIEL BERNSTEIN and CHRISTOPHER LIVINGSTON \\ University of Nebraska, Lincoln, Nebraska 68588
}

\begin{abstract}
An Apple II microcomputer is used for processing observations of human behavior in a longterm live-in laboratory. On-line analysis produces multiple measures of response strength and response pattern for experiments on the reinforcement value of ordinary human activities. An interactive program with an informative screen display monitors observers' data input and operation of the experimental procedures, preventing certain classes of errors. Redundant storage is included to avoid loss of data in the event of equipment failure. The data are handled by humans only one time, as opposed to three with the previous system, reducing both expense and errors. Immediate availability of fine-grained analysis allows more sophistication in withinsubjects designs.
\end{abstract}

The system described here was initiated to reduce the practical problems involved in handling observational data.

\section{LABORATORY SET-UP}

The microcomputer was added to a laboratory that is used for analysis of the reinforcement value of ordinary human activities (e.g., Bernstein \& Ebbesen, 1978). The laboratory is a "standard experimental space" for humans, run continuously in the style of Collier (Collier, Hirsch, \& Kanarek, 1977). Paid experimental subjects are recruited to spend $24 \mathrm{~h} /$ day in a self-contained laboratory apartment for periods of $16-48$ consecutive days. Each subject engages in ordinary human activities, such as reading, sewing, artwork, exercise, playing music, or any other indoor activity or hobby that he or she wishes to bring into the laboratory. The analysis of subjects' behavior is derived from the work of Premack (1965), Timberlake and Allison (1974), and others who have worked on response deprivation. The primary experimental intervention is the restriction of access to the activities that each subject has available in the experiment. Under some conditions, the restriction on access to an activity can be removed if the subject engages in another designated activity. These relationships involve standard instrumental reinforcement contingencies.

The subject's behavior is observed $15 \mathrm{~h}$.of each day.

Development of this program was supported by grants from the University of Nebraska-Lincoln Research Council. Reprint requests should be sent to Daniel Bernstein, 209 Burnett Hall, University of Nebraska, Lincoln, Nebraska 68588. Christopher Livingston is now at Dartmouth College, Hanover, New Hampshire.
A record is kept of the beginning and end of each instance of any of the subject's activities, the location in which the behavior occurs, and environmental or experimental events. The coding system includes a set of parallel systems of categories, such that each system of categories is mutually exclusive and exhaustive. Each system of categories occurs simultaneously with all other systems; for example, the subject may at the same time read a book, sit at a desk, listen to music, and be restricted from doing artwork. Observation is done in 2-h shifts by trained undergraduates. As part of their training, reliabilities are calculated from records of standard videotape segments of behavior; these reliabilities are about .98. The observer is required to keep track of all occurrences of each category and of the restrictions on any activities during experimental conditions and to signal the subject when there are any changes in those restrictions. Details of the observation system are described elsewhere (Bernstein \& Ebbesen, 1978).

\section{DEPENDENT VARIABLES AND DATA ACQUISITION}

\section{Measures}

The recording system notes the exact time of the beginning and end of every event, so it is possible to make several different analyses of the properties of responding. The program calculates two standard measures of response strength: frequency or rate of occurrence and the percentage of total time devoted to each activity (response probability). It is also possible to examine features of the pattern of responding. We can calculate the average duration of each burst or episode of responding and the average interresponse time (IRT), along with a frequency distribution of the individual instances of both duration and IRT. Two other mea- 
sures are calculated: (1) the daily pattern for each activity, the probability of observing each response category during each hour of the observational period, and (2) the sequential pattern of categories, a conditional probability of one activity's following another or the probability of observing a given activity within a certain time following the termination of each other activity. These measures have been used in the analysis of human performance during free-access baseline and under the constraints of reinforcement contingencies (Bernstein \& Ebbesen, 1978). Current reseaich is focusing on the effects of placing constraints on measures of performance other than total duration or frequency.

\section{Old Data Acquisition System}

For 16 subjects observed over a 7-year period, the data were collected using an electromechanical recording system. The observer faced a panel containing a toggle switch for each activity or location category. The switch gated brief pulses (one every $2 \mathrm{sec}$ ) to counters that accumulated the time in 2-sec intervals. Each switch also operated one pen on an Esterline Angus event recorder. Operation of each switch gave both an immediate estimate of the total time devoted to each category and a paper record of the beginning and end of each occurrence of each category. For purposes of running the experimental procedures, only the total amount of time devoted to each category was available; any other analysis of frequency or pattern was dependent upon coding of the event recorder charts. The coding of 30 channels from the event recorder charts was done by hand to a maximum precision of $1 \mathrm{~min}$ (of $900 \mathrm{~min}$ in a total observation period). This hand scoring generally took $3 \mathrm{~h}$ and was typically not completed until the day following the observation. After the event records were scored into numbers, the data were keypunched and put on the disk storage system in the mainframe computer. A preliminary analysis program was then run on the data to eliminate any impossible occurrences or obvious coding errors, and then a series of analysis programs produced data on the measures described above. The keypunching, error check, and program analysis required approximately $5 \mathrm{~h}$, and the task was typically not completed until 2 or 3 days after the data were collected.

\section{New Data Acquisition System}

The laboratory is now equipped with an Apple II Plus $48 \mathrm{~K}$ microcomputer with a single disk drive, a Mountain Hardware calendar clock, a video display unit, and an impact printer. Table 1 shows the screen display, which indicates the current state of the observational code. The observer can readily see which activity the program considers the subect to be engaging in and the physical location. The display also indicates the amount of time each category has been in effect and the total number of seconds since the beginning of this observa- tion period. Upon request, the screen will display the observer's options for changes in categories, changes in locations, or a variety of other special functions. These options are shown at the top of the display in Table 1. When the observer enters the code number for one of the activities listed on the screen, the program first requests that the entry be reconfirmed before it is accepted into the data base. A message is displayed in the center of the screen that defines the entry to be made and requests a press of the return key for confirmation. If confirmation is not given within $5 \mathrm{sec}$, the program assumes the entry was in error and returns to look for another entry. With each accepted entry, the program records both the beginning of a new category and the end of the replaced category. The program also checks for errors involving incorrect en tries (code numbers that are not listed in the menu) or impossible changes, such as attempting to reenter an activity that is already occurring. Specific error messages are displayed on the screen that tell the observer the nature of the error.

Once an entry is accepted, the program begins a chain of functions that disables the keyboard for $6 \mathrm{sec}$. First, each change in the category is sent to a buffer of four events in the computer's memory. The arrival of the latest event bumps the oldest event on to the permanent record of the disk. In addition, the new data entry is immediately included in the summary statistics that provide a description of the performance so far during each observation period. These summary statistics can be examined on the display screen at any time by a command from the keyboard, or the summary statistics can be printed to hard copy by a request from the keyboard. The printing takes approximately $30 \mathrm{sec}$, and it is routinely requested by the observer approximately every $4 \mathrm{~h}$. At the end of each 15-h observation period, the pro-

Table 1

Examples of Screen Display With Observer Options

\begin{aligned} \hline 1) & READ & 2) & GAMES \\ 3) & NDLPT & 4) & TAPES \\ 5) & ART & 6) & EXER \\ 7) & MAINT & 8) & MISC \\ 9) & TABLE & 10) & DESKS \\ 11) & CHAIRS & $12) &$ COUCH \\ 13) & BATH & $14) &$ OTHER \\ 15) & LIST 2 & $16) &$ MONITOR \end{aligned}

2418

$\begin{array}{lrrll}10 / 20 / 81 \quad * & \text { MANUAL } & * & 10: 25.16 & \\ \text { ACTIVITY = READ } & \mathrm{F}=6 & \text { ET }=815 & @ 10: 11.41 \\ \text { LOCATION }=\text { COUCH } & \mathrm{F}=4 & \text { ET }=1200 & @ 10: 05.16 \\ \text { CREDIT }=0 & \text { IRT }=0 & \text { ALLOW }=0 & \text { DSK }=0\end{array}$


Table 2

Example of Display During Contingency Operation

2528

10/20/81 * CONTINGENCY *

$11: 14.25$

ACTIVITY $=$ ART $\mathrm{F}=5$

$\mathrm{F}=12$

CREDIT $=4850$

$\mathrm{IRT}=600$

$\mathrm{ET}=210 @ 11: 10.55$

$\mathrm{ET}=840 @ 11: 00: 25$

ALLOW $=0 \quad$ DSK $=0$

gram prints a complete statistical summary and a listing of each instance of all data entries.

The interactive observation system also automates the restrictions on individual activities and the contingency schedules between pairs of activities. The display on the screen can show the amount of instrumental credit that has been earned and the allowance of time for responses being restricted. These are indicated by the words "credit" and "allowance," respectively, on the screen display shown in Table 2. The display also shows the duration of individual occurrences of each behavior and the required time between instances of the same response category. These are shown in Table 2 under the headings "ET" (elapsed time) and "IRT" (interresponse time). The screen reminds the observer of the necessity of changing conditions when the schedule criteria are met. One of the options listed in the menu allows the experimenter to determine the criterion values of instrumental performance and the ratio of the reinforcement schedule that relates the two activities involved in contingencies. The observers are instructed to keep paper-and-pencil records of all experimental operations so they can be confident about each decision involving a change in the experimental condition. When both the automatic record keeping and individual record keeping suggest that the same decision is to be made, it is extremely unlikely that an error will occur.

\section{Program Printout Format}

Tables 3, 4, and 5 show samples of the three main printouts generated at the end of each day. All of the data in the printouts can be displayed on the screen under the control of the keyboard at any time during the day. At the end of each observation period, a copy is printed that shows the totals and summaries for each day along with a complete listing of the raw data. Table 3 shows a sample from a complete listing of data from a typical day of observation during a baseline condition (no restriction on any activity). All times are given in seconds from the beginning of the observation period, which results in a range of 0 to 54,000 . The listing includes the time during the session when each occurrence begins and ends, the code number of the category or location, and the frequency of occurrences of that category to that time in the day. All other calculations can be derived from this raw data.

Table 4 shows a summary of the descriptive statistics for each activity. For each code number, the printout lists the frequency of occurrence (number of times per $15 \mathrm{~h}$ ), the total number of seconds spent on that activity, the average number of seconds per instance of the category, and the probability of observing each category (calculated by dividing the total duration of that category by the total session time of $54,000 \mathrm{sec}$ ). Following these numbers is a listing of the frequency distribution of the length of individual instances of each category divided in to bins $10 \mathrm{~min}$ in length. For example, a 4 in the first column (labeled B1) means that the subject had four episodes of that response category with a duration under $10 \mathrm{~min}$. This analysis was used for operation of the new experimental procedures, which constrain the pattern of responding. It is also useful in case the average duration (calculated by dividing the total time by frequency) does not represent a typical or characteristic duration. Should there be a bimodal distribution of individual durations, the frequency distribution provides the experimenter a more accurate description of the typical length of an instance of the response category.

Table 5 provides an example of a printout that displays the pattern of occurrence of each activity in each of the $15 \mathrm{~h}$ of observation per day. The numbers in each

Table 3

Portion of Listing of Raw Data From Disk Storage

\begin{tabular}{|c|c|c|c|c|c|c|c|c|c|c|c|}
\hline BEGIN/END & DUR & $\mathrm{FQ}$ & ACT & & $\begin{array}{c}\text { CUM } \\
\text { FREQ }\end{array}$ & BEGIN/END & DUR & $\mathrm{FQ}$ & ACT & & $\begin{array}{c}\text { CUM } \\
\text { FREQ }\end{array}$ \\
\hline $0 / 336$ & 336 & 1 & 14 & $3 / 18 / 81$ & 1 & $0 / 2533$ & 2533 & 1 & 8 & $3 / 18 / 81$ & 2 \\
\hline $336 / 2540$ & 2204 & 1 & 13 & $3 / 18 / 81$ & 3 & $2533 / 2564$ & 31 & 1 & 4 & $3 / 18 / 81$ & 4 \\
\hline $2540 / 2572$ & 32 & 1 & 10 & $3 / 18 / 81$ & 5 & $2564 / 2639$ & 75 & 1 & 7 & $3 / 18 / 81$ & 6 \\
\hline $2572 / 2652$ & 80 & 2 & 14 & $3 / 18 / 81$ & 7 & $2639 / 2663$ & 24 & 2 & 4 & $3 / 18 / 81$ & 8 \\
\hline $2652 / 2685$ & 33 & 2 & 10 & $3 / 18 / 81$ & 9 & $2685 / 4094$ & 1409 & 1 & 9 & $3 / 18 / 81$ & 10 \\
\hline $4094 / 4182$ & 88 & 3 & 14 & $3 / 18 / 81$ & 11 & $4182 / 4772$ & 590 & 2 & 9 & $3 / 18 / 81$ & 12 \\
\hline $4772 / 4844$ & 72 & 3 & 10 & $3 / 18 / 81$ & 13 & $4844 / 4886$ & 42 & 4 & 14 & $3 / 18 / 81$ & 14 \\
\hline $2663 / 5018$ & 2355 & 2 & 8 & $3 / 18 / 81$ & 15 & $5018 / 5058$ & 40 & 2 & 7 & $3 / 18 / 81$ & 16 \\
\hline $5058 / 6744$ & 1686 & 1 & 2 & $3 / 18 / 81$ & 17 & $4886 / 6753$ & 1867 & 3 & 9 & $3 / 18 / 81$ & 18 \\
\hline $6744 / 6825$ & 81 & 3 & 4 & $3 / 18 / 81$ & 19 & $6753 / 6840$ & 87 & 4 & 10 & $3 / 18 / 81$ & 20 \\
\hline $6825 / 9818$ & 2993 & 2 & 2 & $3 / 18 / 81$ & 21 & $9818 / 9863$ & 45 & 3 & 8 & $3 / 18 / 81$ & 22 \\
\hline $6840 / 9844$ & 3004 & 4 & 9 & $3 / 18 / 81$ & 23 & $9844 / 9870$ & 26 & 5 & 14 & $3 / 18 / 81$ & 24 \\
\hline $9863 / 11105$ & 1242 & 1 & 1 & $3 / 18 / 81$ & 25 & $9870 / 11113$ & 1243 & 1 & 11 & $3 / 18 / 81$ & 26 \\
\hline $11105 / 11558$ & 453 & 4 & 4 & $3 / 18 / 81$ & 27 & $11113 / 11562$ & 449 & 5 & 10 & $3 / 18 / 81$ & 28 \\
\hline $11558 / 11653$ & 95 & 4 & 8 & $3 / 18 / 81$ & 29 & $11653 / 12164$ & 511 & 2 & 1 & $3 / 18 / 81$ & 30 \\
\hline $11562 / 11664$ & 102 & 6 & 14 & $3 / 18 / 81$ & 31 & $11664 / 12176$ & 512 & 2 & 11 & $3 / 18 / 81$ & 32 \\
\hline
\end{tabular}


Table 4

Printout of Summary Statistics for Activities for 1 Day

SUMMARY FOR 3/18/81

\begin{tabular}{cclllllllllllll} 
ID & FREQ. & SUM & AVG. & $\%$ & B1 & B2 & B3 & B4 & B5 & OF DURATIONS \\
1 & 11 & 30676 & 2788 & .56 & 1 & 1 & 3 & 1 & 0 & 2 & 0 & 0 & 3 & 0 \\
2 & 6 & 13448 & 2241 & .24 & 0 & 0 & 4 & 0 & 1 & 0 & 1 & 0 & 0 & 0 \\
3 & 0 & 0 & 0 & 0 & 0 & 0 & 0 & 0 & 0 & 0 & 0 & 0 & 0 & 0 \\
4 & 13 & 1982 & 152 & .03 & 13 & 0 & 0 & 0 & 0 & 0 & 0 & 0 & 0 & 0 \\
5 & 0 & 0 & 0 & 0 & 0 & 0 & 0 & 0 & 0 & 0 & 0 & 0 & 0 & 0 \\
6 & 0 & 0 & 0 & 0 & 0 & 0 & 0 & 0 & 0 & 0 & 0 & 0 & 0 & 0 \\
7 & 7 & 2236 & 319 & .04 & 6 & 0 & 1 & 0 & 0 & 0 & 0 & 0 & 0 & 0 \\
8 & 7 & 5658 & 808 & .10 & 5 & 0 & 0 & 1 & 1 & 0 & 0 & 0 & 0 & 0 \\
\hline
\end{tabular}

Table 5

Printout of Pattern of Use Across the Day for Activities

\section{DAILY PATTERN FOR 3/18/81}

\begin{tabular}{|c|c|c|c|c|c|c|c|c|c|c|c|c|c|c|c|c|}
\hline ACT & HOUR & 1 & 2 & 3 & 4 & 5 & 6 & 7 & 8 & 9 & 10 & 11 & 12 & 13 & 14 & 15 \\
\hline 1 & & 0 & 0 & 26 & 66 & 92 & 20 & 98 & 100 & 85 & 90 & 60 & 93 & 49 & 0 & 0 \\
\hline 2 & & 0 & 57 & 72 & 0 & 0 & 0 & 0 & 0 & 0 & 0 & 0 & 0 & 47 & 95 & 99 \\
\hline 3 & & 0 & 0 & 0 & 0 & 0 & 0 & 0 & 0 & 0 & 0 & 0 & 0 & 0 & 0 & 0 \\
\hline 4 & & 1 & 2 & 0 & 27 & 7 & 0 & 2 & 0 & 7 & 0 & 0 & 0 & 2 & 3 & 0 \\
\hline 5 & & 0 & 0 & 0 & 0 & 0 & 0 & 0 & 0 & 0 & 0 & 0 & 0 & 0 & 0 & 0 \\
\hline 6 & & 0 & 0 & 0 & 0 & 0 & 0 & 0 & 0 & 0 & 0 & 0 & 0 & 0 & 0 & 0 \\
\hline 7 & & 2 & 1 & 0 & 0 & 0 & 78 & 3 & 0 & 0 & 9 & 39 & 0 & 0 & 0 & 0 \\
\hline 8 & & 96 & 39 & 1 & 5 & 0 & 0 & 0 & 0 & 7 & 0 & 0 & 6 & 0 & 0 & 0 \\
\hline
\end{tabular}

column are calculated by dividing the total number of seconds during which an activity occurred within that hour by 3,600 . Should an activity have a consistently high probability of occurrence during some periods of the day and a consistently low probability in others, this information might be used in the running of the contingency operation. None of the data displayed in Tables 4 and 5 , with the exception of the total duration in seconds, was previously available during the experiment. These data were available only after 2 or 3 days of hand analysis and computer processing. With these data available immediately, it is possible to use the more complicated descriptions of performance to guide experimental procedures.

\section{PROGRAM FORMAT}

The program is written in APPLESOFT BASIC, and it requires no special modification of the language or the hardware. The program is structured with a logic section that directs the flow to subroutines that perform functions such as reading the clock, changing the screen display, storing data on the disk, and changing the summary statistics in the active memory. Regardless of the screen display option selected, the program continues to read the clock many times each second and accumulates time in the appropriate categories coded by the observer. The program gives the observer access to all features of the computer's memory but does not inter- rupt the basic gathering of information about the subject's activity and location.

\section{Safeguards Against Data Loss}

When equipment is used for the collection of data over long sessions, there is always the danger that equipment failure might result in the loss of data for an entire observation period. Also, a very slow machine might add error variance to observations by keeping the keyboard inactive while processing an entry. This has not been a problem with the present equipment and program, for the functions are generally quite fast, including writing data to the disk. No automatic function of the observation procedure will keep control away from the keyboard longer than $6 \mathrm{sec}$, and this level of precision is quite acceptable for our purposes. The total session length each day is over $54,000 \mathrm{sec}$, and the typical length of an instance of the response categories we study is well over $500 \mathrm{sec}$. The print option, which generates hard-copy summaries during the observation session, is used only at times when the subject's behavior is extremely likely to be stable (e.g., when he or she is in the bathroom or has just begun an activity that typically lasts for long periods of time). Even though control is away from the keyboard for up to $30 \mathrm{sec}$ during printing, the judgment of the observers has been quite good, and we have yet to lose any data entries as a result of running a printout during a session.

The essence of the data collection system is the 
redundancy of representation of the data. A complete summary of all descriptive statistics of the data collected up to any point in the day is kept in the computer's memory. The raw data are also written to the disk with each entry, so there is both an active memory and a permanent memory representation in the electronic part of the observation and recording system. While it is extremely unlikely that an equipment failure or unusual surge of power could wipe out both the current memory in the machine and all the information written on the disk, we still have the paper copy generated by the print option at periodic times during the day, so even that catastrophic event would never lose more than 2 or $3 \mathrm{~h}$ of data.

Another potential difficulty with a computer system is that a program or input error might cause the program to stop running, thus losing the data in memory when the program is restarted. Even though this would not result in the loss of data from the permanent memory record on the disk storage, it would make a combination of two separate records necessary, as the program is written to consider each start-up of the program to be the beginning of an entirely new session. To avoid this problem, we have taken advantage of the APPLESOFT BASIC statement ON ERROR GOTO, which allows the program to continue to run while informing the observer that an error has occurred. The observer is instructed to copy by hand the data found in the four buffers displayed at the bottom of the screen, since these data would be lost in the event that the program should come to a stop. For example, this feature keeps the program from crashing when there is a mechanical problem with the transfer of data to or from the disk. So far, in over 70 days of observation, no data have been lost due to equipment failure. This high rate of reliability is due, at least in part, to the fact that very few data are in active memory at any time. The memory contains summaries calculated from the raw data and only the four most recent entries; the bulk of the raw data are stored on the disk. The present version of the program with an entire day's data stored and summarized uses less than half of the available memory in the central processing unit.

\section{ADVANTAGES OF THE NEW SYSTEM}

The most immediate result of using this system is a dramatic reduction in the time and cost required to produce a printout of each day's observation. The complete analysis is performed by the machine, stored on disk, and printed on paper in about $2 \mathrm{~min}$. In addition to saving money and time by reducing the number of times that the data are handled by researchers, there are no additional errors added into the data base from repeated human processing.

The second major advantage derives from the observation system's immediate analysis of the observer's entry. Given the nature of the coding and recording system, there are certain entries that cannot be made. The observation program catches these errors and asks the observer to correct them before they enter the data base. In addition, the double check on each entry makes sure that simple typographic errors do not get into the data, since the observer must reconfirm each entry before it is accepted.

A third advantage is derived from the very fast availability of the detailed analyses, such as the distribution of durations of the individual instances of each category. It has become possible to do experiments using finegrained data in planning the exact nature of the experimental procedures. The research questions currently under study involve descriptions of both the molar quantitative measures of performance that are available immediately and the detailed analyses of patterns of responding that are less obvious to a first-order analysis. In the past, it was possible to analyze these data only after the fact, so it was not possible to manipulate any patterns of performance as an independent variable in the experiment. In addition, since the data processing takes place so quickly, it has become practical to undertake additional detailed post hoc analyses of the data that were prohibitively expensive when the work had to be done by hand.

Fourth, the program also provides an independent check on the observer's handling of the experimental procedures themselves, making it far less likely that an observer will make an error in the carrying out of the detailed restrictions and contingencies that are necessary for the experimental analysis and reinforcement value. While errors of this type have not been common in the past, the likelihood that such an error would occur has been dramatically reduced. Finally, the system has made training of observers much easier and much less time-consuming. With very fast tumaround of the analysis of each observer's test record of videotape samples, it is possible to check the reliability of each observer and to provide immediate feedback. Given a constant amount of time and resources for training, this means that each observer receives more training before the beginning of the observation period. The new system is far less complicated than the old data acquisition system, and the experimental procedures part of the program is more self-explanatory.

\section{RITERENCDS}

Berngtein, D., \& Ebbesen, E. Reinforcement and substitution in humans: A multiple-response analysis. Journal of the Experimental Analysis of Behavior, 1978, 30, 243-253.

Collier, G., Hirsch, E., \& Kanarek, R. The operant revisited. In W. Honig \& J. Staddon (Eds.), Handbook of operant behavior. New York: Prentice-Hall, 1977.

Premack, D. Reinforcement theory. In D. Levine (Ed.), Nebraska Symposium on Motivation. Lincoln: University of Nebraska Press, 1965.

Timberlake, W., \& Allison, J. Response deprivation: An empirical approach to instrumental performance. Psychological Review, 1974, 81, 146-164. 